\title{
Die Krise in der Krise - Subjektive Wahrnehmungen und Reaktionsmuster von Beschäftigten
}

Karina Becker/Ulrich Brinkmann/Oliver Nachtwey

\begin{abstract}
Die Finanzkrise ist öffentlich vor allem als ein Phänomen aus der Welt der Banken und Versicherungen diskutiert worden, das erhebliche gesamtwirtschaftliche Auswirkungen zeitigt. Sie wirft allerdings auch Fragen nach dem Krisenbewusstsein, Krisenerleben und den Krisenreaktionen der Beschäftigten auf. Nehmen sie eine betriebliche Realität passiv hin, die ihnen immer weitere Zugeständnisse für das vage Versprechen der Arbeitsplatzsicherung abverlangt? In dem Beitrag wird gezeigt, dass Krise für viele Beschäftigte seit zwei Dekaden ein Zustand der Normalität ist, dass sich aber an Langfristfolgen wie dem Gesundheitsschutz neue Handlungsmöglichkeiten für betriebliche Akteure entwickeln können.
\end{abstract}

\section{Einleitung}

Es ist offenkundig, dass die subjektive Wahrnehmung von Krisen eng an ihre Auswirkungen gekoppelt ist. Anders als die längerfristige Krise der Beschäftigtengesundheit, die wir in diesem Aufsatz näher beleuchten wollen, scheint die „Finanzmarktkrise“ bei vielen Beschäftigten noch nicht angekommen zu sein, ihre Konsequenzen drohen eher ,über Bande“; man betrachtet sie wie ein Gewitter in der Ferne, von dem man hofft, dass es an einem vorbeizieht. Eine Erklärung dafür ist ihre (staatliche) Abfederung beispielsweise durch den verstärkten Einsatz von Kurzarbeit, aber auch die betriebliche Externalisierung der Krisenfolgen (z.B. Entlassung von Leiharbeitern). Zu Beginn der aktuellen „Finanzmarktkrise“ tat sich die deutsche Bundesregierung zunächst durch Herunterspielen oder das rhetorische Verschieben der Probleme nach Übersee hervor, bevor sie sich schließlich zu makroökonomischen und -politischen Aktionen durchrang. Seither stabilisiert sie mit Bürgschaften, Quasi-Verstaatlichungen und Deficit-Spending die Wirtschaft. Allein die Ausgaben zur Stützung des Bankensektors betragen mit knapp $100 \mathrm{Mrd}$ € in den Jahren 2007-2009 mehr als die Hälfte des Schuldenzuwachses - Bürgschaften nicht eingerechnet, ${ }^{1}$ übertroffen noch von der deutschen Beteiligung an der Stützung des Euro. Der Staat hat sich durch die Politik eines „regulierten Liberalismus“ (Watkins 2010) - allen neoliberalen Unkenrufen zum Trotz - als entscheidende Stabilisie- rungsinstanz eines aus den Fugen geratenen Finanzmarktkapitalismus erwiesen.

Insolvenzen auf breiter Basis wurden zwar vermieden, allerdings schlägt sich die Krise in einer neuen Restrukturierungswelle und einer Reihe von Zugeständnissen der Belegschaften nieder. Schon vor Krisenbeginn gestalteten sich viele betriebliche Pakte immer weniger als reziproker Tausch (Jürgens/Krzywdzinski 2006); seit Beginn der Finanzmarktkrise häufen sich „krisenkorporatistische“ (Hans-Jürgen Urban) Lösungen und betriebliche „painsharing agreements“ (Marginson 2010), bei denen vor allem die Stammarbeitskräfte Zugeständnisse bei Arbeitsbedingungen und Lohn (Bispinck et al. 2010) im Tausch gegen die Zusicherung des Erhaltes von Arbeitsplätzen machen.

Gleichwohl sind vielerorts Beschäftigte bereits seit mehr als einer Dekade an einen vom Management forcierten Krisendiskurs gewöhnt, der von immer neuen Rationalisierungswellen und Flexibilisierungsmaßnahmen begleitet wird. Krise ist für sie kein Ausnahmezustand (dieser ist erst bei Insolvenz oder der eigenen Entlassung erreicht), Krise ist für viele vielmehr eine Alltagserfahrung, geradezu betriebliche Normalität (Dörre et al. 2009). ${ }^{2}$ Dies gilt speziell für Gesundheitsfragen, die zudem in ostdeutschen Betrieben besonders virulent sind, da dort seit zwei Dekaden das Damoklesschwert der Dauerkrise die betrieblichen Restrukturierungen bestimmt (Brinkmann 2002).

Das Phänomen der Krise als Normalität werden wir nachfolgend am Beispiel der Beschäftigtengesundheit diskutieren. $\mathrm{Zu}$ diesem Zweck entwickeln wir zunächst das zugrundeliegende Verhältnis von kapita- listischer Entwicklung und betrieblichem Arbeits- und Gesundheitsschutz (Abschnitt 2). Anschließend zeigen wir, wie mit der langen Krise der Weltwirtschaft und der Shareholder-Value-Steuerung der Unternehmen Arbeits- und Gesund-

\footnotetext{
http://www.bundesbank.de/download/presse/ pressenotizen/2010/20100419.maastrich.php.

2 Unsere eigenen Erhebungen durch Experteninterviews in drei regionalen Kliniken im Mai und Juni 2010 haben ergeben, dass die Finanzkrise für die betrieblichen Orientierungen, Interessen und Handlungsstrategien bislang fast keine Rolle spielt. Einen vertiefenden Einblick gibt der dargestellte Krankenhausfall in diesem Beitrag (Abschnitt 4.2). Eine bisher unveröffentlichte Projektskizze vom soziologischen Forschungsinstitut (SOFI) Göttingen und vom Institut für Sozialwissenschaftliche Forschung (ISF) München kommt zu ähnlichen Ergebnissen.
}

Karina Becker, Dr., Soziologin, wissenschaftliche Mitarbeiterin am Lehrstuhl Arbeits-, Industrie- und Wirtschaftssoziologie der Friedrich-Schiller-Universität Jena. Arbeitsschwerpunkte: Finanzmarktkapitalismus, KMU, betriebliche Mitbestimmung, Arbeits und Gesundheitsschutz. e-mail: Karina.Becker@uni-jena.de Ulrich Brinkmann, Prof., Dr., lehrt Wirtschaftssoziologie der Universität Trier. Arbeitsschwerpunkte: Finanzmarktkapitalismus, Gewerkschaften, Beteiligung, Arbeits- und Gesundheitsschutz, Unternehmensreorganisation. e-mail: Brinkmann@uni-trier.de Oliver Nachtwey, Dr., Ökonom, wissenschaftlicher Mitarbeiter am Lehrstuhl Wirtschaftssoziologie der Universität Trier. Arbeitsschwerpunkte: Arbeits- und Industrie soziologie, Gewerkschaften und Parteien, vergleichende Kapitalismusforschung. e-mail: Nachtwey@uni-trier.de 
heitsschutz in Form und Inhalt reduziert wurden (Abschnitt 3). Anhand von drei betrieblichen Fallstudien erläutern wir abschließend, wie Beschäftigte auf die lange Krise der Beschäftigtengesundheit vor der Folie der aktuellen Finanzmarktkrise reagieren (Abschnitt 4). Bei den Fallstudien ${ }^{3}$ handelt es sich wegen ihrer anhaltenden Krisenbetroffenheit um ostdeutsche Betriebe (Fahrzeugindustrie, Fall A; Maschinenbau, Fall B; Gesundheitswesen, Fall C), in denen Beschäftigte versuchen, ihre Ansprüche an den Arbeits- und Gesundheitsschutz nicht hinter anderen potenziellen - zum Teil auch inszenierten - Krisenfolgen zurückzustellen. Vielmehr lässt sich vor allem in den ersten beiden Fällen beobachten, dass Beschäftigte unter bestimmten Bedingungen Strategien des Erhalts ihrer Gesundheit entwickeln. Hier - so argumentieren wir im Fazit (Abschnitt 5) - liegen Chancen für kollektives Handeln der Arbeitnehmer.

\section{Vom faulen Kompromiss zum Bruch des fordisti- schen Versprechens}

Die Krise der Beschäftigtengesundheit könnte in der Begrifflichkeit des bekannten HIRES-Reports einer europäischen Expertengruppe geradezu das „Ausmaß einer Pandemie" annehmen (Kieselbach et al. 2009, S. 18). Dies ist auch darauf zurückzuführen, dass die Langzeiteffekte betrieblicher Restrukturierungen für die Gesundheit von Beschäftigten bislang kaum in den Blick genommen wurden (ebd.).

Analysiert man Krisenursachen von Finanzmarkt und Gesundheit, so lassen sich eine Reihe von Gemeinsamkeiten ausmachen: Die Gründe liegen in den wachsenden Problemen des Kapitalverwertungsprozesses seit den 1970er Jahren - insbesondere in den geschaffenen Produktionsüberkapazitäten der global agierenden Industriezweige (Brenner 2009; Harvey 2010). Hinzu kam der Wandel in der politischen Ökonomie der Wohlfahrtsstaaten, durch den öffentliche Daseinsvorsorge und öffentliche Güter entweder privatisiert oder ökonomisiert wurden (Harvey 2005).

Die Jagd nach profitablen Kapitalanlagemöglichkeiten führte einerseits zu den Verwerfungen im Banken- und Versicherungssektor des Finanzmarktkapita- lismus - mit allen Folgen wiederum für die Realwirtschaft. Auf der Suche nach Möglichkeiten, die Renditen auf das eingesetzte Kapital zu verbessern, finden sich andererseits auch im produzierenden und nicht-finanziellen Dienstleistungsgewerbe selbst seit einigen Jahren neue Strategien zur „Optimierung“ der Unternehmensprozesse und Arbeitsorganisation, die in der Regel weitreichende Restrukturierungsprozesse nach sich ziehen. Es sind diese Prozesse permanenter Reorganisation, die bei den Betroffenen sowohl Unsicherheit erzeugen als auch die zentralen Ursachen der Gesundheitskrise in den Betrieben darstellen.

Historisch betrachtet ist die Verschiebung der Grenze zwischen Kommodifizierung und De-Kommodifizierung der Arbeitskraft von Beginn an eine der zentralen Konfliktlinien im Kapitalismus. Während die Unternehmer vor allem auf eine hinreichende Reproduktion von geeigneten Arbeitskräften angewiesen sind, war für die Beschäftigten und die Gewerkschaften der Schutz des individuellen Arbeitsvermögens ein zentrales betriebliches und überbetriebliches Kampffeld. Aufgrund erfolgreicher Kämpfe und technischwissenschaftlichen Fortschritts schien es lange Zeit nur die eine vorgezeichnete mögliche Entwicklung - eine Erhöhung des Schutzniveaus - zu geben. Voraussetzung war dabei stets, dass sich Akteure zu einem machtvollen kollektiven Handeln für die Ausweitung der Schutzrechte stark machten. Unter den Bedingungen des Nachkriegsfordismus setzte sich diese Tendenz fort. Das kurze „Goldene Zeitalter" brachte zahlreiche Verbesserungen in der Entwicklung des Arbeits- und Gesundheitsschutzes mit sich - auch hier waren es Beschäftigte, Betriebsräte und Gewerkschaften, denen dies gelang, indem sie ihre strukturellen, organisationalen und institutionellen Machtzugänge verbreitern konnten. Mehr noch: Man kann mit Fug und Recht behaupten, dass spätestens seit dieser Phase eine schützenswerte Beschäftigtengesundheit zu den Elementen des zeitgenössischen Rechtfertigungssystems des Kapitalismus gehört.

Allerdings: Trotz dieses offenkundigen Trends zur Absicherung der Arbeitskraft gegen die Risiken des Marktes (Hobsbawm 1995, S. 356ff.) bestand dessen Grundlogik weiter fort. Denn während in der Frühphase des Industriekapitalismus ein Raubbau an der eigenen Gesundheit im Tausch ge- gen Überleben sichernde Arbeit wie selbstverständlich zum Alltag der abhängigen Beschäftigten gehörte, verschwand dieser betriebliche Handel mit der Beschäftigtengesundheit im Fordismus nicht von der Bildfläche, sondern kehrte durch die Hintertüre zurück, erfuhr einen Formwandel - wenn auch auf weit höherem Niveau dank institutioneller Absicherungen, gesetzlicher Regulierungen, wissenschaftlichen Fortschritts und vorbildlicher Praxis. Denn Bestandteil dieses „Handels“ war auf der einen Seite die Verbesserung der materiellen Teilhabe, auf der anderen Seite die Inkaufnahme gesundheitsbelastender Arbeitsbedingungen in der fordistischen Massenproduktion. Bei allen Verbesserungen schloss dieser fordistische Deal aus der Perspektive des Arbeits- und Gesundheitsschutzes (AGS) daher immer schon einen faulen Kompromiss ein, führte er doch bei den Beschäftigten trotz relativer Verbesserungen im AGS zur sukzessiven Dezimierung ihrer Gesundheitssubstanz.

Dieser Tausch wurde dann von der Unternehmensseite mit dem Übergang zum Finanzmarktkapitalismus vielerorts mehr oder weniger explizit aufgekündigt (vgl. dazu Becker et al. 2007). Die Folgen sind weitreichend und mittlerweile vielfach beschrieben. Für den AGS bedeutet dies die Verlängerung und Flexibilisierung der Arbeitszeiten, die Intensivierung der Arbeitskraftvernutzung, die Prekarisierung der Arbeitsverhältnisse, die Heterogenisierung von Arbeits- und Werkverträgen, die Wertsteuerung von Arbeit in den Unternehmen (um nur einige Punkte zu nennen) sowie die Gefahr einer Umkehr des langfristigen Trends der Verbesserung von Arbeitsbedingungen. Deren Folgen lassen sich zurzeit noch nicht präzise messen; allerdings ist die drastische Zunahme psychischer Erkrankungen ein deutliches Indiz. Mehr als jeder zehnte Arbeitsunfähigkeitstag ist auf sie zurückzuführen. Parallel dazu nehmen auch die „klassischen“ körperlichen Belastungen wieder zu (vgl. zu beidem die Ergebnisse der PARGEMAWSI-Betriebsrätebefragung 2008/2009 im Schwerpunktheft der WSI-Mitteilungen 7/2010).

3 Die Betriebe wurden von der Autorin und den Autoren in verschiedenen Forschungsprojekten intensiv begleitet und beforscht. Das empirische Material (Befragungen und Interviews), auf das sich der Beitrag stützt, wurde zwischen 2008 und 2010 erhoben. 
Dieser betriebliche Wandel entwickelte sich in einem Umfeld, in dem zwar noch mit dem Arbeitsschutzgesetz von 1996 eine verbesserte gesetzliche Grundlage für den betrieblichen Arbeits- und Gesundheitsschutz geschaffen wurde (z.B. durch die Institutionalisierung der Gefährdungsbeurteilung). In der Folge aber stand und steht ein legislativer Kahlschlag unter dem Siegel der „Entbürokratisierung“ auf der Tagesordnung bis hin zur geradezu skelettierten Arbeitsstättenverordnung. Bestandteile dieses Umfeldes sind auch gewandelte gesellschaftliche und betriebliche Diskurse. Nicht die Verbesserung problematischer Arbeitsverhältnisse, sondern der Erhalt der eigenen Beschäftigungsfähigkeit durch gesundheitsförderliches Verhalten steht heute hoch im Kurs - dies schon deshalb, weil betrieblicher Arbeits- und Gesundheitsschutz als Kostenfaktor neu definiert wird und damit in Rechtfertigungsnöte stürzt.

\section{Kostensenkung zur Wertsteigerung: Gesund- heit auf der Kippe}

Indem sich der Shareholder-Value-Gedanke (Rappaport 1986; Dörre 2002) aus dem begrenzten Umfeld börsengehandelter Unternehmen zu einem gesamtwirtschaftlichen Leitbild verallgemeinerte, gewann er auch eine hohe Bedeutung für die Praxis des betrieblichen Gesundheitsschutzes: In stilbildenden Unternehmen wird dieser heute ebenfalls als cost-center geführt, dessen Anreizstrukturen die Experten dazu anhalten, möglichst wenig Kosten zu produzieren, um damit zur Steigerung des Unternehmenswertes beizutragen. Diese Kostensenkungsstrategie wird zwar als „Optimierung" bezeichnet, faktisch führt sie jedoch zu einer spürbaren Absenkung von Standards im AGS, wie betroffene Sicherheitsfachkräfte und Beschäftigte in Interviews und Gesprächen berichten.

Damit schreibt sich verstärkt fort, was sich durch den punktuellen Einsatz von prekär Beschäftigten bereits seit geraumer Zeit andeutet: Denn auch hinsichtlich des Gesundheitsschutzes tut die von Holst et al. (2009) skizzierte Disziplinierung durch den strategischen Einsatz von Leiharbeit ihr Übriges. Viele Leiharbeiter sehen sich gezwungen, problematische
Arbeitsbedingungen in Kauf zu nehmen oder krank zur Arbeit zu erscheinen, was sich nolens volens wiederum als Präsentismusverhalten auch bei den Stammarbeitskräften niederschlägt. Deutlich wird heute: Auf den faulen Kompromiss im Fordismus folgte im betrieblichen AGS der offene Bruch des fordistischen Versprechens im heutigen Finanzmarktkapitalismus. Krisenförmige Zuspitzungen fungieren dabei zusätzlich als Katalysatoren. In Krisen drückt sich der Widerspruch zwischen gesellschaftlicher Produktion und privatkapitalistischer Aneignung deutlich aus (vgl. MEW Bd. 20, S. 257). Crouch (2010) und auch Baccaro (2010, S. 347) haben deshalb die Frage aufgeworfen, wie sich dieser Widerspruch manifestiert bzw. an welchem Ort und auf welche Weise Krisenbetroffene ihrem Unmut freien Lauf lassen.

Während die Finanzmarktkrise aus den dargelegten Gründen bislang wenig Ansatzpunkte für betriebliches Handeln zu bieten scheint, sieht dies bei der beschriebenen Krise der Beschäftigtengesundheit anders aus, denn hier findet keine zeitliche und räumliche Entkopplung von Ursachen und Wirkungen statt - dies gilt im Übrigen auch für betriebliche Problemlösungsstrategien.

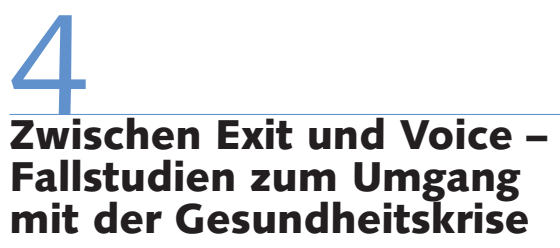

Allerdings lassen sich verschiedenartige Reaktionsweisen von Beschäftigten im Umgang mit der eigenen Gesundheit beobachten; die gängige, also auch mehrheitliche Antwort vieler befragter Beschäftigter besteht in der forcierten Fortschreibung einer Strategie der Unterordnung der Gesundheit unter betriebswirtschaftliche Zwänge mit dem Effekt der verstärkten Vernutzung ihres Arbeitsvermögens. Es finden sich aber auch empirische Hinweise, dass eine Krisenverdopplung, also ein Hinzutreten der Finanzkrise zur Gesundheitskrise, keineswegs zwangsläufig zu einer verschärften (Selbst-)Unterwerfung der Beschäftigten unter das Postulat der Verwertung der eigenen Gesundheit führt.

In diesen Fällen beginnen Beschäftigte zum Teil das Verhältnis ihrer Rollen als Mitglieder eines Unternehmens einerseits und Teilnehmer des Arbeitsmarktes ande- rerseits neu zu definieren (Fall A und C). Beteiligungsorientierte Strategien können die Diskurse des Managements zurückdrängen (Fall B).

Wir haben die Betriebsfälle vor dem Hintergrund der Doppelkrise von Ökonomie und Beschäftigtengesundheit im Hinblick auf drei Dimensionen untersucht:

- Handelt es sich bei der Krisenreaktion der Beschäftigten um situative oder nachhaltig ausgerichtete Aktionen?

- Sind sie geprägt von individuellen oder kollektiven Handlungsansätzen?

- Finden wir - in Hirschmans (1970) Terminologie gesprochen - vor allem Exit(auf das Verlassen der Situation orientierte) oder Voice-Strategien (auf Veränderung über Interessenartikulation orientiert) vor?

Die nachfolgenden Fallstudien stellen lediglich Momentaufnahmen dar; sie sind nicht repräsentativ, lassen sich aber durchaus danach befragen, inwieweit sie eine Erfolg versprechende Praxis für betriebliche und gewerkschaftliche Interessenvertreter in einem schwierigen Umfeld sein können.

\subsection{FALL A: RESSOURCEN- ORIENTIERTER UMGANG MIT DER GESUNDHEITSSUBSTANZ}

Die Beschäftigten des ersten Betriebsfalls sind unmittelbar mit den Auswirkungen der Finanzkrise konfrontiert. Es handelt sich um ein Unternehmen der Fahrzeugindustrie, dem zum Zeitpunkt der Erhebung die krisenbedingte Schließung drohte. Der Standort beschäftigt rund $1500 \mathrm{Ar}$ beitskräfte und weist im konzerninternen Benchmarking eine hohe Produktivität auf, die auf jahrelange innerbetriebliche Umstrukturierungen zurückzuführen ist. Für die Beschäftigten - darunter einige hundert Leiharbeiter - bedeutete die an Effizienzkriterien ausgerichtete Optimierung der Produktionsabläufe in erster Linie einen Wegfall von abpuffernden Reserven sowie eine hohe Verdichtung der Arbeitsabläufe. Im Tausch dafür erhielt die Belegschaft allerdings nicht die erhoffte (Arbeitsplatz-)Sicherheit, sondern war mit einem fortdauernden Drohszenario konfrontiert, das bis heute anhält und das durch widersprüchliche Krisendiskurse der Konzernleitung noch geschürt wird. Die Betriebsräte berichten, dass diese langjährigen Erfahrungen zu einer deutlichen Disziplinierung der Beschäftigten geführt 
Tabelle 1: Arbeitsplatzangst und Priorität von Arbeits- und Gesundheitsschutz - in \% -

\begin{tabular}{lcccc}
\hline & \multicolumn{4}{c}{$\begin{array}{c}\text { Zustimmung und Ablehnung zu der Aussage: } \\
\text { "Solange der Arbeitsplatz sicher ist, kann man auch einen }\end{array}$} \\
& mangelhaften Gesundheitsschutz akzeptieren." \\
& Ja & Teilweise & Nein & Summe \\
\hline besorgt um eigenen Arbeitsplatz & 3,1 & 5,5 & 91,4 & 100 \\
teilweise besorgt um eigenen Arbeitsplatz & 0,0 & 7,7 & 92,3 & 100 \\
nicht besorgt um eigenen Arbeitsplatz & 2,0 & 6,9 & 91,1 & 100 \\
\hline Beschäftigtenbefragung im Unternehmen A im Jahr 2009, N=653, & & & \\
Skalenniveau aggregiert. & & & W S MITTEILUNGEN \\
Quelle: Erhebung der Autoren.
\end{tabular}

haben, zu einer Internalisierung der Kommodifizierung und zu einem Rückschrauben der Ansprüche an Arbeitssicherheit und Gesundheitsschutz. In dieser Dominanz marktförmiger Exit-Ausrichtung des Unternehmens liefen Partizipationsinitiativen der Beschäftigten im Rahmen eines regelmäßigen Verbesserungsprozesses ins Leere. Seit der Krankenstand über dem Konzerndurchschnitt lag, versuchte das Management, diesen durch die systematische Durchführung von „Krankenrückkehrgesprächen“ zu drücken - zur marktzentrierten gesellte sich damit eine verstärkte hierarchisch-machtförmige Disziplinierung der Belegschaft.

Auch der seit 1996 gesetzlich vorgeschriebenen Pflicht, psychische Belastungen im Rahmen einer Gefährdungsbeurteilung zu erfassen und Maßnahmen zu deren Minimierung abzuleiten, kam die Geschäftsführung nicht nach. Initiativen des Betriebsrats in den Blick zu nehmen, scheiterten am Widerstand des Managements. Um Handlungsdruck aufzubauen, führte der Betriebsrat Anfang 2009 eine aktivierende Beschäftigtenbefragung durch. Diese Strategie war in doppelter Hinsicht erfolgreich: Zum einen dokumentierte sie erstmals systematisch die psychischen Belastungen der Beschäftigten; zum anderen erfüllte die Befragung eine Informationsfunktion, indem sie über die gesetzlich vorgeschriebenen AGS-Standards aufklärte und gegen deren Unterlaufen mobilisierte. Der angesichts der angespannten Situation hohe Rücklauf von fast $50 \%$ deutet darauf hin, dass trotz zunehmender Krisenbetroffenheit die Bereitschaft der Beschäftigten zur Thematisierung gesundheitlicher Belastungen keineswegs sank. Darauf verweist auch die verstärkte Inanspruchnahme von sogenannten Überlastanzeigen in der Zeit nach der Befragung - ein Instrument, das der Betriebsrat seit einigen Jahren den Beschäftigten anbietet, um Probleme des
Leistungs- und Zeitdrucks systematischer bearbeiten zu können. „Jeden Tag kommt jetzt mindestens eine Überlastanzeige“, so ein Betriebsratsmitglied. „Da spielt auch bei vielen der Frust rein: ,Ich werde hier verschlissen und weiß gar nicht, ob ich hier in drei Monaten noch einen Arbeitsplatz habe, wenn es das Unternehmen nicht mehr gibt ““. In dieser Haltungsänderung deutet sich ein Orientierungswandel an: Die Tatsache, möglicherweise in naher Zukunft „wieder auf dem Markt zu sein“, führt offenbar zum Umdenken. Die Perspektive der Beschäftigten beschränkt sich nicht mehr allein auf das Unternehmen, sondern nimmt den Arbeitsmarkt in den Blick. Damit wenden sie sich geradezu aus Not der Exit-Option zu. Die drohende Betriebsschließung führt bei diesen Beschäftigten dazu, dass sie sich verstärkt über ihre eigenen Marktoptionen Gedanken machen. Ein Beschäftigter schilderte diesen Reflexionsprozess wie folgt: „Na ja, den Kollegen wird natürlich gerade bei dieser Riesendiskussion, die jetzt im Gange ist, im Zusammenhang mit der Krise, denen wird natürlich auch immer mehr bewusst: ,Das einzige Gut, was ich noch habe, ist meine Gesundheit. Und wenn die erstmal kaputt ist, na, dann habe ich ja gar nichts mehr.' Das muss auch jedem klar sein. Wir können nur noch Bandarbeit nach all den Jahren hier im Werk. Wo sollen wir denn sonst hin, wenn das hier den Bach runtergeht?"

Diese Reaktionsweisen deuten darauf hin, dass die Beschäftigten der Gefahr der Arbeitslosigkeit mit einem ressourcenschonenden Umgang mit der eigenen Gesundheit begegnen. Der Erhalt der eigenen Beschäftigungsfähigkeit (vgl. exemplarisch Ulich/Wülser 2009 sowie Pickshaus/ Spieker 2009) wird zur entscheidenden Maßgabe. Das drohende Szenario, das Unternehmen verlassen zu müssen, zieht auch eine Erosion des Commitments der Beschäftigten dem Unternehmen gegen- über nach sich - eine Form des inneren Exits: „Ich habe mich über Jahre hier abgerackert und jetzt steht man vielleicht von einem Tag auf den anderen einfach so auf der Straße. Meine Motivation ist zurzeit gleich null“ (nicht freigestelltes Betriebsratsmitglied).

Der erhöhte Stellenwert der Ressource Gesundheit im Bewusstsein der Beschäftigten spiegelt sich auch in den Befragungsergebnissen wider: Statt krisenbedingt „draufzuzahlen" und Gesundheitsressourcen im Gegenzug zu einer vermeintlichen Sicherung von Arbeitsplätzen zu investieren, sind über $90 \%$ der Befragten nicht oder eher nicht bereit, einen mangelhaften Gesundheitsschutz zu akzeptieren - und dies weitgehend unabhängig davon, ob sich die Befragten um ihren Arbeitsplatz bereits sorgen oder nicht, wie Tabelle 1 zeigt.

Dass Beschäftigte auch unter verschärften Krisenbedingungen auf ihre Gesundheit achten, belegt dazu der Krankenstand, der entgegen dem üblichen Trend nicht zurückgeht. „Das zeigt uns, dass die Leute wirklich fertig sind. Die können nicht mehr. Die können nicht mal mehr krank zur Arbeit kommen“" (BR-Mitglied). Stattdessen nimmt die Zahl der Anträge auf Frühverrentung zu, was sich auch dadurch erklärt, dass die Beschäftigten Sorge haben, die - bei einer Insolvenz gefährdeten - über Jahre erworbenen Ansprüche und Einzahlungen zu verlieren.

Neben dieser eher auf die eigene Person bezogenen veränderten Handlungsorientierung lassen sich in diesem Betriebsfall auch Strategien eines nachhaltigen Umgangs mit der Gesundheit ausmachen, die auf kollektive Voice setzen: So erfüllte die vom Betriebsrat durchgeführte Befragung - wie erwähnt - eine Sensibilisierungs-, Informierungs- und Mobilisierungsfunktion der Belegschaft für die Gesundheitsthematik, die von weiteren, auf verschiedenen Ebenen ansetzenden Voice-Strategien (Betriebszeitungen und -versammlungen, Aushänge etc.) flankiert wurden. Die Befragungsergebnisse legten offen, dass die hohen Fallzahlen von Fehlbelastungen nicht allein auf individuelle Probleme einzelner zurückgeführt werden können, sondern auf unter arbeitswissenschaftlicher Perspektive dysfunktionale Organisationsstrukturen und Arbeitsorganisation, die Folge eines marktzentrierten Managementansatzes sind. Darauf verweisen auch die zahlreichen offenen Nennungen, in denen der Führungsstil als „Management by 
Überforderung" bezeichnet und kritisiert wird, dass „Ideen ausgebremst [werden], angeblich aus Kosten- und Kapazitätsgründen“. Der Betriebsrat schuf sich mit diesem Beteiligungsinstrument die Möglichkeit, in den innerbetrieblichen Aushandlungen mit dem Management auf der Basis abgesicherter Daten Gesundheitsbelastungen der Beschäftigten zu bündeln und zu artikulieren.

Für den Betriebsrat erwies sich die Durchführung einer aktivierenden Befragung in der Krise als EmpowermentStrategie, die darin mündete, dass er ein Jahr nach der drohenden Schließung eine Betriebsvereinbarung zur Durchführung einer Gefährdungsbeurteilung psychischer Belastungen abschloss.

\subsection{FALL B: BETEILIGUNGSORIEN- TIERTE GEFÄHRDUNGSBEURTEILUNG}

Der Fall eines Maschinenbauunternehmens führt vor Augen, dass Beschäftigte durch den Rückgriff auf kollektive VoiceStrategien ihre Arbeitsbedingungen selbst unter schwierigen ökonomischen Bedingungen nachhaltig gestalten und damit maßgeblich verbessern können. Dafür wurde ein Weg eingeschlagen, der es dem Management auch in Krisenzeiten erschwert, Arbeitssicherheits- und Gesundheitsprobleme zu ignorieren: Dazu ein Statement des Betriebsratsvorsitzenden: „Arbeits- und Gesundheitsschutz wurde in den letzten Jahren zum Thema gemacht. Es wurde ins Bewusstsein gerufen und auch über Verstöße berichtet, ohne jetzt strafen zu wollen, sondern das einfach sichtbar machen. (...) Gesundheit ist wirklich ein Thema geworden. Das kriegt man jetzt nicht mehr weg. Das hat mit Krise und Kurzarbeit überhaupt nichts mehr zu tun." Das diesem Vorgehen zugrunde liegende Instrument ist die im Arbeitsschutzgesetz verankerte Gefährdungsbeurteilung, die den Beschäftigten und dem Betriebsrat weitgehende Mitbestimmungsrechte einräumt $(\$ \$ 5,3,6)$. Darüber hinaus gewährleistet das Betriebsverfassungsgesetz ( $\$ 87$ Abs. 1 Satz 7) das Mitbestimmungsrecht von Betriebsräten bei „Regelungen über die Verhütung von Arbeitsunfällen und Berufskrankheiten sowie über den Gesundheitsschutz im Rahmen der gesetzlichen Vorschriften oder der Unfallverhütungsvorschriften“. Das bloße Vorhandensein dieser gesetzlich garantierten Möglichkeiten stellt jedoch noch nicht sicher, dass diese auch in Anspruch genommen werden, wie der defizitäre Umsetzungsstand der Gefährdungsbeurteilung eindrücklich zeigt. Im hier vorgestellten Beispiel bildeten sie jedoch das Fundament für einen aufwendigen, durch einige Hürden und konfrontative Auseinandersetzungen mit dem Management gezeichneten Weg der Umsetzung der gesetzlichen Vorgaben. Dabei entschied sich das die Gefährdungsbeurteilung initiierende Betriebsratsgremium für einen besonders anspruchsvollen, auf Langfristigkeit und Kontinuität angelegten Ansatz: Die Beschäftigten sollten auf allen Stufen - der Erfassung, Ermittlung und Maßnahmenableitung - konsequent beteiligt werden. Die erfolgreiche Umsetzung dieser Strategie trug in hohem Maße zur Nachhaltigkeit des Prozesses bei-auch, als die Finanzkrise in Form von Auftragseinbrüchen das Unternehmen erreichte. Denn die betriebliche Verankerung des Themas auf der Beschäftigtenebene erschwerte es dem Management, diesem mit Verweis auf die Krise den Handlungsdruck abzusprechen und den Promotoren damit die Legitimität zu entziehen.

Das beteiligungsorientierte Vorgehen trägt auch dem Modernisierungsgedanken des novellierten Arbeitsschutzgesetzes Rechnung (Beschäftigte als Experten ihrer eigenen Gesundheit). Ausschlaggebend für ein beteiligungsorientiertes Vorgehen im Arbeits- und Gesundheitsschutz waren die positiven Erfahrungen des Betriebsratsvorsitzenden bei der Erarbeitung eines neuen Arbeitszeitmodells. Dabei stützte er sich auf das Betriebsverfassungsgesetz ( $\$ 80$ Abs. 2 Satz 3), das regelt, dass Betriebsräte sachkundige Arbeitnehmer zur Unterstützung ihrer Arbeit heranziehen können, die für diese Zeit von ihrer Arbeit freigestellt werden und dabei dem Benachteiligungs- und Kündigungsschutz unterliegen. Der nunmehr eingeschlagene Weg einer systematischen Beteiligung ist in verschiedener Hinsicht ein Fortschritt: So machen die Beschäftigten die Erfahrung der Selbstwirksamkeit. Die abgeleiteten Maßnahmen zur Verbesserung des Arbeits- und Gesundheitsschutzes wurden nicht wie lange Zeit üblich den Beschäftigten von oben übergestülpt, vielmehr werden sie von den Beschäftigten getragen und auch selbst kontrolliert. Hinzu kommt, dass sich der Betriebsrat des Rückhalts für seine Arbeit versichert, was sich bei der nachfolgenden Betriebsratswahl, aber auch in den Auseinandersetzungen mit dem Management auszahlte, welches die Finanzkrise mit dem mittlerweile schon geläufigen Argument „Wir haben jetzt keine Zeit für solche Themen“ nutzen wollte, um den Prozess zu blockieren. Für die Beschäftigten war der Auftragsrückgang jedoch kein Argument, die sich über die letzten Jahre angestauten Arbeitsschutzprobleme, deren Folgen sie bereits in Form gesundheitlicher Verschleißerscheinungen unmittelbar erlebten, zurückzustellen. Derart mobilisiert wurde die Verbesserung des Arbeits- und Gesundheitsschutzes zu ihrem Thema, dessen Bearbeitung der Betriebsrat gegen den Widerstand des Managements durchsetzte.

\subsection{FALL C: KOMMODIFIZIERUNG DER EIGENEN ARBEITSKRAFT}

Eine anders gelagerte Strategie, auf unter Gesundheitsaspekten problematische Arbeitsbedingungen zu reagieren, lässt sich in der Intensivpflege eines Krankenhauses beobachten. Die Rahmenbedingungen in diesem Bereich sind insofern untypisch, als die Beschäftigten aufgrund des Fachkräftemangels in dieser Region auf einem Angebotsmarkt agieren. Vergleichbar mit anderen Unternehmen ist die Implementierung marktzentrierter Steuerungsformen im Krankenhaus durch neue prospektive Finanzierungsarten, wie Budgets, Kopf- und Fallpauschalen (vgl. Kühn 2003; Simon 2001). Im Zuge des aktuellen Umbruchs zum Marktkapitalismus halten damit Wettbewerbsprinzipien in einem Sektor Einzug, in dem sich medizinische Sachverhalte und Probleme vorrangig an außerökonomischen, ethischen Maßstäben orientierten.

Wie in den beiden Fällen zuvor äußern sich die Folgen dieser Entwicklung bei den Beschäftigten in Form einer sich abzeichnenden, in einigen Fällen bereits virulenten Gesundheitskrise: Die Pflegekräfte gaben an, dass ihre Arbeit in den letzten Jahren durch eine drastisch steigende Arbeitsintensivierung geprägt ist (zu vergleichbaren Ergebnissen kommen auch Böhlke et al. 2009). Die Versorgung der Patienten kann nur noch durch den permanenten Einsatz von Leiharbeitskräften gewährleistet werden (vgl. auch Bräutigam et al. 2010); stetig zunehmender Zeit- und Leistungsdruck bestimmen den Arbeitsalltag. Hinzu kommt, dass die Beschäftigten im Branchenvergleich unterdurchschnittlich bezahlt werden. Ein 
Pfleger erläuterte die daraus resultierende Unzufriedenheit wie folgt: „Man kann nicht jemanden im Dreischichtsystem, zwölf Tage am Stück, davon fünf Nächte, arbeiten lassen und dann geht der mit eineinhalb Scheinen nach Hause - schon gar nicht in der Intensivpflege, wo man auch häufig Arzt-Arbeiten macht. Das geht einfach nicht. Das ist menschenunwürdig, das macht einen fertig. " $\mathrm{Zu}$ diesen ohnehin schon prekären Bedingungen kommt noch ein ausgeprägtes Anerkennungsdefizit der Pflegekräfte, das die Folge einer wahrgenommenen mangelnden Wertschätzung ihrer Arbeit ist.

Die Unausgewogenheit zwischen Leistung und Entlohnung verdichtet sich zu auf Dauer krank machenden Gratifikationskrisen (Siegrist 1996), wie die steigende Zahl von Burnout-Fällen in der Pflege dokumentiert. Alle interviewten Pflegekräfte (Durchschnittsalter ca. 30 Jahre) gaben an, dass sie ihre Tätigkeit im reorganisierten Krankenhaus ihrer Gesundheitssubstanz beraubt und sie diese Arbeit keinesfalls bis zum Rentenalter ausüben können: „Auf der Intensivstation wird man nicht alt, das hält keiner lange durch." Viele von ihnen reduzieren ihre Arbeitszeit, nur so „kann man dem Arbeitsdruck standhalten. Es geht hier immerhin um die Versorgung von Patienten. Da hat man ja auch eine hohe Verantwortung."

Neben dieser Variante des Erhalts von Beschäftigungsfähigkeit um den Preis der Verminderung eines ohnehin schon geringen Einkommens greifen einige - vor allem jüngere - Pflegekräfte auf eine Individualstrategie zurück, mit der es ihnen gelingt, kurzfristig ihre „Vermarktungsbedingungen “ zu maximieren. Dazu bieten sie auf Basis von Teilselbstständigkeit ihre Arbeitskraft zusätzlich zur regulären Beschäftigung als Angestellte des gleichen Krankenhauses an. Mit dieser SelbstKommodifizierung erwirtschaften sie pro Schicht das Drei- bis Vierfache des Gehalts eines normalen Arbeitstags als Krankenhauspflegekraft. Das Marktrisiko für diese „Unternehmer in eigener Sache“ ist, aufgrund der günstigen Arbeitsmarktlage und ihrer zusätzlichen Festanstellung im Krankenhaus, begrenzt. Der Anreiz ist indes groß, sich einem erhöhten Gesundheitsrisiko durch eine deutliche Verlängerung der Arbeitszeiten auszusetzen: Ein Pfleger, der neben seiner Vollzeitstelle noch auf Honorarbasis zusätzliche Schichten übernimmt, schilderte sein Handeln wie folgt: „Wenn mal eine Schicht nicht so stressig war und ich denke, eine weitere ist noch drin, lass ich mich buchen. Das geht schon mal." An dieser Praxis lässt sich eine individuelle Bewirtschaftung der eigenen Gesundheit ablesen, bei der kurzfristige Interessen vor den langfristigen Erhalt der Gesundheit und damit der Arbeitskraft gestellt werden. Sie repräsentiert einen Beschäftigtentyp, der sich als „Gesundheitsmanager“ (vgl. Becker et al. 2008) bezeichnen lässt. Gesundheit ist auch in dieser Perspektive eine handelbare Ressource - mit allen Gefahren eines frühzeitigen Verschleißes derselben. Da sich dieser Tausch kurzfristig finanziell auszahlt, wird dieses Vorgehen sowohl im Kollegenkreis als auch von der Stationsleitung nicht nur toleriert, sondern auch unterstützt: „Wenn ich jetzt jemanden fragen muss, ob er einspringt, weil jemand krank ist und ich weiß, dass der nebenbei selbstständig ist, sage ich dem ,Lass dich für morgen früh buchen'. Das ist einfach attraktiver. Man bringt ja auch keinen mehr dazu, einzuspringen für einen feuchten Händedruck " (Leiterin einer Intensivstation). Die Teilselbstständigkeit dieser Pflegekräfte stellt eine zumindest partielle Umstellung der Beschäftigten auf die Exit-Logik dar: Man bietet sich aufgrund der Unzufriedenheit auf einem eigens geschaffenen Markt in genau jener Nische zur Miete an, in der man ohnehin eingesetzt worden wäre - mit dem Unterschied, dass aufgrund der günstigen Marktkonstellation die Entlohnungsbedingungen deutlich besser sind.

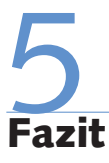

Der vorliegende Beitrag geht von der Überlegung aus, dass sowohl der akuten Finanzmarktkrise als auch der schwelenden Krise der Gesundheit von Beschäftigten eine ähnliche Ursachenkonstellation zugrunde liegt, deren Wurzeln in den Kapitalverwertungsproblemen im Finanzmarktkapitalismus zu suchen sind. Dies hat dazu geführt, dass vielerorts Krisendiskurse und Umstrukturierungen zur betrieblichen Normalität geworden sind. Im Unterschied zur jüngsten Finanz- und der darauf folgenden Wirtschaftskrise, die allerdings aufgrund staatlicher Interventionen in weiten Bereichen kaum durchgeschlagen ist, ist die Gesundheits- krise zwar ebenso ein betriebliches Alltagsphänomen, aber sie ist direkt „am eigenen Leib " erfahrbar und bedroht das eigene Arbeitsvermögen. Diese Erfahrbarkeit birgt indes nicht nur Risiken, sondern eröffnet auch Chancen für betriebliche Reaktionsweisen. Deren Bandbreite wurde anhand von drei Fällen aufgezeigt, wobei das besondere Augenmerk auf Fragen nach der spezifischen Qualität der Handlungsvarianten gelegt wurde: Handelt es sich primär um kollektive oder individuelle Strategien, sind sie langfristig oder situativ angelegt und folgen sie der Exit- oder der Voice-Logik?

In Fall A hat der jahrelange Druck des Unternehmens auf den AGS zu einer Umorientierung der Beschäftigten auf ihre Beschäftigungsfähigkeit auf dem externen Arbeitsmarkt geführt. In Fall C gab die Arbeitsmarktsituation den Beschäftigten einen Anreiz, Risiken in der eigenen Beschäftigtengesundheit einzugehen. Als problematisch erwiesen sich die auf eine Marktlogik setzenden Exit-Strategien unabhängig davon, ob sie aus einer Position der Stärke (Anbietermarkt im Pflegebereich) oder der Schwäche (fatalistische Abkehr vom Unternehmen) herrührten für die Voice-Fähigkeit der betrieblichen Interessenvertreter: In beiden Fällen liefen sie tendenziell dem Präventionsgedanken im AGS zuwider und unterminierten kollektive, auf Voice setzende Strategien.

Dass vor allem langfristig angelegte, kollektive, auf Voice setzende Strategien eine nachhaltige Bearbeitung der Gesundheitsprobleme bis hin zur Prävention ermöglichen, zeigen die Ansätze des Betriebsrates in Fall A und der Fall B. Indem die damit in Gang gesetzten, auf Beteiligung basierenden Prozesse der Verbesserung und Verstetigung des Arbeits- und Gesundheitsschutzes von den beteiligten Beschäftigten getragen werden, gilt das Nachhaltigkeitsargument auch unter den Bedingungen der Finanzkrise. Mobilisierte, für ihre eigene Gesundheitskrise sensibilisierte Beschäftigte fügen sich offenbar nicht ohne Weiteres dem Krisendiskurs des Managements, wonach der AGS der Unternehmensrendite nachgeordnet werden kann. Es war den Interessenvertretern zumindest in Ansätzen gelungen, im Gesundheitsschutz eine kollektive Haltelinie gegen Restrukturierungsprogramme $\mathrm{zu}$ setzten, die die unmittelbaren (Gesundheits-)Interessen der Beschäftigten zur Grundlage macht. 
Baccaro, L. (2010): Does the global financial crisis mark a turning point for labour?, in: Socio-Economic Review 2, S. 341-348

Becker, K./Brinkmann, U./Engel, T. (2007): Die Haut auf dem Markte. Betrieblicher Gesundheitsschutz im Marktkapitalismus, in: PROKLA, Zeitschrift für kritische Sozialwissenschaft 148, S. 383-401

Becker, K./Brinkmann, U./Engel, T. (2008): Beschäftigte als „Unternehmer der eigenen Gesundheit" ?, in: Bogedan, C./Müller-Schoell, T./ Ziegler, A. (Hrsg.): Demografischer Wandel als Chance. Erneuerung gesellschaftlicher Solidaritätsbeziehungen?, Hamburg, S. 173-192 Bispinck, R./Dribbusch, H./Fikret, Ö. (2010): Auswirkungen der Wirtschaftskrise auf die Beschäftigten, in: WSI-Report 2 (März), Düsseldorf Böhlke, N./Gerlinger T./Mosebach, K./Schmucker, R./Schulten, T. (2009): Privatisierung von Krankenhäusern. Erfahrungen und Perspektiven aus der Sicht der Beschäftigten, Hamburg

Bräutigam, C./Dahlbeck, E./Enste, P./Evans, M./Hilbert, J. (2010): Flexibilisierung und Leiharbeit in der Pflege - eine explorative Studie, Arbeitspapier der Hans-Böckler-Stiftung 215, Düsseldorf, im Erscheinen Brenner, R. (2009): What is good for Goldman Sachs is good for America - The origins of the current crisis, Center for Social Theory and Comparative History, UCLA (18 April), Los Angeles

Brinkmann, U. (2002): Umbruch von unten? Betriebliche Akteure in der ostdeutschen Transformation, München/Mering

Brinkmann, U./Dörre, K. (2006): Die neue Unternehmerkultur Zum Leitbild des "Intrapreneurs" und seinen Implikationen, in: Brinkmann, U./Krenn, K./Schief, S. (Hrsg.): Endspiel des Kooperativen Kapitalismus? - Institutioneller Wandel unter den Bedingungen des marktzentrierten Paradigmas, Wiesbaden, S. 136-168

Crouch, C. (2010): The financial crisis a new chance for labour movements? Not yet, in: Socio-Economic Review 2, S. 353-357

Dörre, K. (2002): Kampf um Beteiligung. Arbeit, Partizipation und industrielle Beziehungen im flexiblen Kapitalismus, Wiesbaden Dörre, K./Behr, M./Eversberg, D./Schierhorn, K. (2009): Krise ohne Krisenbewusstsein? Zur subjektiven Dimension kapitalistischer Landnahmen, in: PROKLA, Zeitschrift für kritische Sozialwissenschaft 157, S. 559-576

Fuchs, T./Trischler, F. (2007): Arbeitsqualität aus Sicht von Erzieherinnen und Erziehern. Ergebnisse aus der Erhebung zum DGB-Index Gute Arbeit, http://www.inifes.de/publikationen.htm
Harvey, D. (2005): A short History of Neoliberalism, Oxford Harvey, D. (2010): The Enigma of Capital and the Crisis of Capitalism, London

Hirschman, A. O. (1970): Exit, Voice, and Loyalty. Responses to Decline in Firms, Organizations and States, Cambridge/London

Hobsbawm, E. (1995): Das Zeitalter der Extreme. Weltgeschichte des 20. Jahrhunderts, München/Wien

Holst, H./Nachtwey, O./Dörre, K. (2009): Funktionswandel von Leiharbeit. Neue Nutzungsstrategien und ihre arbeits- und mitbestimmungspolitischen Folgen, Frankfurt

Jürgens, U./Krzywdzinski, M. (2006): Globalisierungsdruck und Beschäftigungssicherung - Standortsicherungsvereinbarungen in der deutschen Automobilindustrie zwischen 1993 und 2006, in: WZB Discussion Paper SP III 2006-303, Berlin

Kieselbach, T. et al. (2009): Gesundheit und Restrukturierung. Innovative Ansätze und Politikempfehlungen, München/Mehring

Kühn, H. (2003): Ethische Probleme der Ökonomisierung von Krankenhausarbeit. Dienstleistungsqualität und Qualität des Arbeitslebens im Krankenhaus, in: Büssing, A./ Glaser, J. (Hrsg.): Dienstleistungsqualität und Qualität des Arbeitslebens im Krankenhaus, Göttingen, S. 77-98 Marginson, P. (2010): New forms of co-operation, new forms of conflict, in: Socio-Economic Review 2, S. 360-364

Pickshaus, K./Spieker, H. (2009): Beschäftigungsfähigkeit herstellen und entwickeln - Ansprüche aus gewerkschaftlicher Sicht, in: Zeitschrift für Arbeitswissenschaft 4, S. 285-290

Rappaport, A. (1986): Creating shareholder value, New York Schröder, L./Urban, H. (2009): Gute Arbeit. Handlungsfelder für Betriebe, Politik und Gewerkschaften, Frankfurt

Siegrist, J. (1996): Soziale Krisen und Gesundheit, Göttingen

Simon, M. (2001): Die Ökonomisierung des Krankenhauses. Der wachsende Einfluss ökonomischer Ziele auf patientenbezogene Entscheidungen, Berlin

Skrabs, S./Wegener, A. (2010): Trotz Krise: Tariflicher Gesundheitsschutz im Sozial- und Erziehungsdienst, in: WSI-Mitteilungen 7, S. 382-384

Ulich, E./Wülser, M. (2009): Gesundheitsmanagement in Unternehmen, Wiesbaden

Watkins, S. (2010): Shifting Sands, in: New Left Review 1, S. 5-28 